MPP-2010-109

\title{
Phenomenology of SUSY SU(5) with type I+III seesaw
}

\author{
Carla Biggid * and Lorenzo Calibb团 \\ Max-Planck-Institut für Physik (Werner-Heisenberg-Institut), D-80805 München, Germany
}

\begin{abstract}
We consider a supersymmetric SU(5) model where two neutrino masses are obtained via a mixed type I+III seesaw mechanism induced by the component fields of a single SU(5) adjoint. We have analyzed the phenomenology of the model paying particular attention to flavour violating processes and dark matter relic density, assuming universal boundary conditions. We have found that, for a seesaw scale larger than $10^{12 \div 13} \mathrm{GeV}, \operatorname{BR}(\mu \rightarrow e \gamma)$ is in the reach of the MEG experiment in sizable regions of the parameter space. On the other side, current bounds on it force $\operatorname{BR}(\tau \rightarrow \mu \gamma)$ to be well below the reach of forthcoming experiments, rendering thus the model disprovable if a positive signal is found. The same bounds still allow for a sizable positive contribution to $\epsilon_{K}$, while the $\mathrm{CP}$ violation in the $B_{s}$ mixing turns out to be too small to account for the di-muon anomaly reported by the D0 collaboration. Finally, the regions where the neutralino relic density is within the WMAP bounds can be strongly modified with respect to the constrained MSSM case. In particular, a peculiar coannihilation region, bounded from above, can be realized, which allows us to put an upper bound on the dark matter mass for certain set-ups of the parameters.
\end{abstract}

\section{INTRODUCTION}

Neutrino masses are an indication for the presence of new physics beyond the standard model (SM). The simplest extension consisting in adding to the SM fields three right-handed $(\mathrm{RH})$ neutrinos and giving them a Dirac mass is not very satisfactory since it would require extremely small Yukawa couplings, much smaller then the ones for charged particles. A nice way of explaining neutrino masses as well as their smallness is through the socalled seesaw mechanism: new fields with masses much heavier than the electroweak (EW) scale, once integrated out, generate a dimension-five operator which, after EW symmetry breaking, gives a Majorana mass to neutrinos. When neutrino masses are generated at tree-level, three heavy mediators are possible, namely singlet fermions (corresponding to the so-called type-I seesaw [1]), triplet scalar (type II [2]) and triplet fermions (type III [3] $)^{1}$. Independently of the nature of the mediators, the neutrino mass results to be suppressed by their heavy mass. Interestingly, $\mathcal{O}(1)$ Yukawa couplings require the scale of new physics to be around the grand unification scale. It is then natural to study the seesaw mechanisms in the context of grand unified theories (GUT). Moreover, as it is well known, the presence of low scale supersymmetry (SUSY) triggers the unification of gauge couplings, so that usually SUSY GUTs are considered.

In the literature plenty of models of SUSY GUTs including a seesaw mechanism for neutrino mass generation has been proposed. Actually, many different GUTs have been studied, involving the different seesaws, especially the types I and II. As for the type III (and type I+III), its embedding in a SUSY GUT was firstly proposed in Ref. [5] and then discussed in Ref. [6] (in the context of a

\footnotetext{
*Electronic address: biggio@mppmu.mpg.de

${ }^{\dagger}$ Electronic address: calibbi@mppmu.mpg.de

${ }^{1}$ See Ref. [4] for a review on the three mechanisms.
}

renormalizable model) and in Ref. [7], and embedded in a flavour model in Ref. [8]. In all these cases the grand unified group considered is $\mathrm{SU}(5)$, which is the simplest group in which the SM gauge group can be embedded (for a wide discussion about SUSY SU(5) with the three different seesaw mechanisms, see [9]). In a minimal version of $\mathrm{SU}(5)$ [10] neutrinos are massless, so that this GUT model has to be extended in order to account for neutrino masses. The model we consider here is somehow the simplest extension of a SUSY SU(5) accounting for neutrino masses, since with the simple addition of only one $\mathrm{SU}(5)$ representation two neutrino masses are generated via a mixed type I+III seesaw mechanism [7]. This is different from other models, where at least two representations were needed to account for two or three neutrino masses. Indeed, in the case of type I seesaw, the addition of at least two singlets is mandatory, while for the type II one must include both a triplet and its conjugate (or, in terms of SU(5) multiplets, a $\mathbf{1 5}$ and a $\overline{15}$ 11] $)^{2}$. This model is the SUSY version of the model firstly proposed in Ref. [12, 13]. Also there two neutrino masses are obtained via a mixed type I+III seesaw, but being the model non-SUSY, the spectrum is completely different. Indeed, to guarantee unification, the triplet must be at the TeV scale, while the singlet mass is not specified. In this model lepton flavour violation (LFV) is usually suppressed either by the small Yukawas or by a large mass. This is the standard situation in non-SUSY seesaw models, unless a low-scale inverse seesaw is realized [4, 14]. Furthermore, in presence of cancellations in the neutrino mass matrix, sizable rates of LFV processes are still possible (see for example, in the context of type I+III seesaw, [15]).

Here we study in detail the phenomenology of this "minimal" SUSY SU(5) with massive neutrinos, paying

\footnotetext{
2 Also in the type III models mentioned before more representations are introduced, either matter [8] or Higgs [6].
} 
particular attention to the effects on LFV processes. In order to isolate the effects purely induced by the RG running of the SUSY parameters we assume universal soft masses at the GUT scale. We also discuss possible related effects in the quark sector and how the region of the parameter space where the lightest SUSY particle (LSP) is a viable dark matter (DM) candidate are modified. The rest of the paper is organized as follows: in Sect. II we introduce the model, in Sect. III we discuss the flavour violation, in Sect. IV] we present numerical results and in Sect. $\nabla$ we conclude. The renormalization group equations (RGEs) of this model are gathered in the Appendix.

\section{THE MODEL}

We consider a SUSY SU(5) model where the matter content is enlarged with a $\mathbf{2 4}$ representation of $\mathrm{SU}(5)$, in order to get neutrino masses. Indeed the $\mathbf{2 4}$ can be decomposed under $\mathrm{SU}(3)_{c} \times \mathrm{SU}(2)_{L} \times U(1)_{Y}$ as $S+T+$ $O+V+\bar{V}$, where

$$
S \sim(1,1)_{0} \quad T \sim(1,3)_{0} \quad O \sim(8,1)_{0} \quad V \sim(3,2)_{-5 / 6} .
$$

$S$ and $T$ give mass to two neutrinos via a mixed type I+III seesaw mechanism. The relevant superpotential terms are then

$$
\begin{aligned}
W_{S U(5)}= & \frac{1}{4}\left(Y_{10}\right)_{i j} \mathbf{1 0}_{i} \mathbf{1 0}_{j} \mathbf{5}_{H}+\sqrt{2}\left(Y_{\overline{5}}\right)_{i j} \mathbf{1 0}_{i} \overline{\mathbf{5}}_{j} \overline{\mathbf{5}}_{H}+ \\
& y_{24}^{i} \overline{\mathbf{5}}_{i} \mathbf{2 4} \mathbf{5}_{H}+\frac{M_{24}}{2} \mathbf{2 4} \mathbf{2 4}+\cdots
\end{aligned}
$$

Here $\overline{\mathbf{5}}=\left(d^{c}, L\right), \mathbf{1 0}=\left(u^{c}, Q, e^{c}\right)$ and $\overline{\mathbf{5}}_{\mathbf{H}}=\left(H_{T}, H_{1}\right)$, as usual. Below the $\mathrm{SU}(5)$ breaking scale, the superpotential reads:

$$
\begin{aligned}
W= & \left(Y_{u}\right)_{i j} u_{i}^{c} Q_{j} h_{u}+\left(Y_{d}\right)_{i j} d_{i}^{c} Q_{j} h_{d}+\left(Y_{e}\right)_{i j} e_{i}^{c} L_{j} h_{d}+ \\
& y_{T}^{i} L_{i} T h_{u}+y_{S}^{i} L_{i} S h_{u}+y_{V}^{i} d_{i}^{c} V h_{u}+ \\
& \frac{M_{s}}{2} S S+\frac{M_{T}}{2} T T+\frac{M_{O}}{2} O O+M_{V} V \bar{V}
\end{aligned}
$$

Notice that, from Eq. (1), it follows that the $S U(3)$ octet $O$ and the field $\bar{V}$ do not have Yukawa interactions with fields which remain lighter than the GUT scale.

From Eq. (2), it is easy to see that the singlet $S$ and the neutral component of the triplet $T$ generate neutrino masses via a seesaw mechanism:

$$
m_{\nu}=-\frac{v_{u}^{2}}{2}\left(\frac{y_{S}^{i} y_{S}^{j}}{M_{S}}+\frac{y_{T}^{i} y_{T}^{j}}{M_{T}}\right) .
$$

Notice that, since $S$ and $T$ belong to the same $\mathrm{SU}(5)$ multiplet, at the GUT scale $\vec{y}_{T}=\sqrt{10 / 3} \vec{y}_{S}=\vec{y}_{24} / \sqrt{2}$. The previous $m_{\nu}$ is then a rank- 1 matrix and only one neutrino mass is generated. In principle the Yukawas could be misaligned in the running from the GUT scale to the seesaw scale simply via RGE effects, but in practice the resulting misalignment is too small, giving rise to a second neutrino mass much smaller that the solar mass. It is then clear that the GUT relation on the Yukawa couplings must be somehow altered, in order to get two massive neutrinos. Since non-renormalizable operators should anyway be added to the SUSY SU(5) lagrangian in order to break the unwanted relation $Y_{e}=Y_{d}^{T}$ which is in disagreement with the experimental value of fermion masses $[16]^{3}$, we can also add non-renormalizable operators involving the $\mathbf{2 4}[12]$ :

$$
\begin{aligned}
W_{\mathrm{NR}} \supset & \frac{1}{\Lambda}\left[y_{1}^{i}\left(\overline{\mathbf{5}}_{i} \mathbf{2 4}\right)\left(\mathbf{2 4}_{\mathbf{H}} \mathbf{5}_{\mathbf{H}}\right)+y_{2}^{i}\left(\overline{\mathbf{5}}_{i} \mathbf{2} \mathbf{4}_{\mathbf{H}}\right)\left(\mathbf{2 4} \mathbf{5}_{\mathbf{H}}\right)+\right. \\
& \left.y_{3}^{i} \overline{\mathbf{5}}_{i} \operatorname{tr}(\mathbf{2 4 \mathbf { 2 4 }} \mathbf{H}) \mathbf{5}_{\mathbf{H}}\right],
\end{aligned}
$$

where $\Lambda$ represents the cut-off of the theory (e.g. the Planck scale $M_{\mathrm{Pl}}$ ) and $\mathbf{2 4}_{\mathbf{H}}$ is the Higgs in the adjoint representation which breaks $\mathrm{SU}(5)$, with $\left\langle\mathbf{2 4}_{\mathbf{H}}\right\rangle=\operatorname{diag}(1 / 3,1 / 3,1 / 3,-1 / 2,-1 / 2) \sqrt{3 / 5} v_{\mathrm{G}}$. The three above operators could be for instance generated by integrating-out, respectively: a Higgs superfield in the $\mathbf{4 5}+\overline{45}$ representation, a matter $\mathbf{4 5}+\overline{\mathbf{4 5}}$, a $\mathrm{SU}(5)$ singlet, all having a mass of the order of the scale $\Lambda$.

After SU(5) breaking, the Yukawa couplings of the different components of the $\mathbf{2 4}$ split and the matching at the GUT scale reads:

$$
\begin{aligned}
& \vec{y}_{T}=\frac{1}{\sqrt{2}} \vec{y}_{24}+\frac{1}{2} \sqrt{\frac{3}{10}} \frac{v_{\mathrm{G}}}{\Lambda}\left(\vec{y}_{1}+\vec{y}_{2}\right), \\
& \vec{y}_{S}=\sqrt{\frac{3}{20}} \vec{y}_{24}+\frac{3}{20} \frac{v_{\mathrm{G}}}{\Lambda}\left(\vec{y}_{1}+\vec{y}_{2}\right)+\frac{1}{2} \frac{v_{\mathrm{G}}}{\Lambda} \vec{y}_{3}, \\
& \vec{y}_{V}=\frac{1}{\sqrt{2}} \vec{y}_{24}+\frac{1}{2} \sqrt{\frac{3}{10}} \frac{v_{\mathrm{G}}}{\Lambda} \vec{y}_{1}-\frac{1}{2} \sqrt{\frac{2}{15}} \frac{v_{\mathrm{G}}}{\Lambda} \vec{y}_{2} .
\end{aligned}
$$

In practice the coupling $\vec{y}_{3}$ determines the misalignment between $\vec{y}_{S}$ and $\vec{y}_{T}$ that permits to generate two non-zero neutrino masses. As we will see later, LFV processes suggest for the seesaw scale an upper bound around $10^{13 \div 14} \mathrm{GeV}$, corresponding to neutrino Yukawa couplings smaller than $10^{-(1 \div 2)}$. From Eq. (6) we then deduce that $\vec{y}_{3}$ should be $\mathcal{O}(1)$ and the cutoff scale $\Lambda$ cannot be too large, if we want to saturate these bounds. From now on we will consider $\vec{y}_{S}$ and $\vec{y}_{T}$ as independent parameters.

In the same way as the splitting among the Yuwakas is generated via $\mathrm{SU}(5)$ breaking effect, also a splitting in the masses arise. The terms contributing to the masses of the $\mathbf{2 4}$ fields are

$$
\frac{1}{2} M_{24} \operatorname{tr}\left(\mathbf{2 4}^{2}\right)+\lambda_{24} \operatorname{tr}\left(\mathbf{2 4}^{2} \mathbf{2} \mathbf{4}_{\mathbf{H}}\right)+\mathcal{O}\left(\frac{v_{\mathrm{G}}^{2}}{\Lambda}\right),
$$

\footnotetext{
${ }^{3}$ An alternative solution is adding new Higgs representation [17].
} 
giving rise to the following masses:

$$
\begin{aligned}
& M_{S}=\frac{M_{24}}{2}-\lambda_{24} \frac{v_{\mathrm{G}}}{2 \sqrt{60}}+\lambda_{S}^{\mathrm{NR}} \frac{v_{\mathrm{G}}^{2}}{\Lambda}, \\
& M_{T}=\frac{M_{24}}{2}-\lambda_{24} \frac{3 v_{\mathrm{G}}}{2 \sqrt{60}}+\lambda_{T}^{\mathrm{NR}} \frac{v_{\mathrm{G}}^{2}}{\Lambda}, \\
& M_{O}=\frac{M_{24}}{2}+\lambda_{24} \frac{2 v_{\mathrm{G}}}{2 \sqrt{60}}+\lambda_{O}^{\mathrm{NR}} \frac{v_{\mathrm{G}}^{2}}{\Lambda}, \\
& M_{V}=\frac{M_{24}}{2}-\lambda_{24} \frac{v_{\mathrm{G}}}{4 \sqrt{60}}+\lambda_{V}^{\mathrm{NR}} \frac{v_{\mathrm{G}}^{2}}{\Lambda},
\end{aligned}
$$

where the actual expressions of the $\lambda_{X}^{\mathrm{NR}}$ in terms of the possible NR operators couplings can be found in [12].

If we do not allow fine cancellations, we can conclude that the components of the $\mathbf{2 4}$ have masses of the same order of magnitude, so that only small threshold effects are introduced and the successful gauge coupling unification of the MSSM is maintained. Notice that, since LFV processes disfavor a seesaw scale larger than $10^{14} \mathrm{GeV}$, the coupling $\lambda_{24}$ should be $10^{-(1 \div 2)}$ or smaller while the non-renormalizable couplings $\lambda_{i}^{\mathrm{NR}}$ can be larger, depending on the cutoff scale $\Lambda$.

We now focus on neutrino masses. Eq. (3) can be inverted and the Yukawa couplings can be expressed in terms of low energy parameters. Since in this case we have only two massive neutrinos, Casas-Ibarra parameterization is simplified, since the $\mathrm{R}$ matrix depends now only on one complex parameter $z$ 18]. The Yukawa couplings can then be expressed as [13]:

$$
v y_{T}^{i *}=\left\{\begin{array}{lcc}
i \sqrt{M_{T}}\left(U_{i 2} \sqrt{m_{2}^{\nu}} \cos z+U_{i 3} \sqrt{m_{3}^{\nu}} \sin z\right) & \mathrm{NH} \\
i \sqrt{M_{T}}\left(U_{i 1} \sqrt{m_{1}^{\nu}} \cos z+U_{i 2} \sqrt{m_{2}^{\nu}} \sin z\right) & \mathrm{IH}
\end{array}\right.
$$

and

$v y_{S}^{i *}=\left\{\begin{array}{lcc}-i \sqrt{M_{S}} & \left(U_{i 2} \sqrt{m_{2}^{\nu}} \sin z-U_{i 3} \sqrt{m_{3}^{\nu}} \cos z\right) & \mathrm{NH} \\ -i \sqrt{M_{S}}\left(U_{i 1} \sqrt{m_{1}^{\nu}} \sin z-U_{i 2} \sqrt{m_{2}^{\nu}} \cos z\right) & \mathrm{IH}\end{array}\right.$

There is another solution with the opposite sign for the second terms in Eqs. (13, 14). In the above equations, $U_{i j}$ are elements of the PMNS matrix ${ }^{4}$. For the neutrino mass eigenvalues we have in the normal hierarchy $(\mathrm{NH})$ case:

$$
m_{1}^{\nu}=0 \quad m_{2}^{\nu}=\sqrt{\Delta m_{S}^{2}} \quad m_{3}^{\nu}=\sqrt{\Delta m_{A}^{2}+\Delta m_{S}^{2}},
$$

while in the inverted hierarchy ( $\mathrm{IH})$ case neutrino masses are given by:

$$
m_{1}^{\nu}=\sqrt{\Delta m_{A}^{2}-\Delta m_{S}^{2}} \quad m_{2}^{\nu}=\sqrt{\Delta m_{A}^{2}} \quad m_{3}^{\nu}=0,
$$

4 We remind the reader that here, like in the $2 \mathrm{RH}$ neutrinos case, the PMNS matrix only has two phases: a Dirac phase $\delta$ and a Majorana phase $\Phi$. where we take the neutrino mass parameters [21] as measured in the solar and atmospheric oscillation experiments

$$
\begin{aligned}
& \Delta m_{S}^{2} \approx 7.59 \times 10^{-5} \mathrm{eV}^{2}, \\
& \Delta m_{A}^{2} \approx 2.46(2.36) \times 10^{-3} \mathrm{eV}^{2} \quad \mathrm{NH}(\mathrm{IH}) .
\end{aligned}
$$

From Eqs. (13, 14) we see that the Yukawa couplings grow with the square root of the mass of the heavy fermions, as a trivial consequence of the seesaw formula Eq. (3), and increase exponentially when $\operatorname{Im}(z)$ grows. If we want to avoid unnatural cancellations in the neutrino sector (e.g. between the two terms of Eq. (3) $), \operatorname{Im}(z)$ should be $\leq \mathcal{O}(1)$. As we will show later, the present bound on $\mu \rightarrow e \gamma$ can actually constrain it to smaller values.

Notice that in what respect neutrino masses, this model is not different from a model with two righthanded neutrinos (2RHN) [18 20], where for instance the same parameterization of Eqs. (13, 14) holds. However, this model, besides the fact of being better motivated from a GUT perspective, has got some features which distinguish it from a generic $2 \mathrm{RHN}$ model:

- Up to fine-tuning, the parameter space is more restricted, since it is natural to assume $M_{S}$ and $M_{T}$ to be of the same order of magnitude (see Eqs. (9, 10)). Moreover, barring cancellations, $y_{S}^{i}$ and $y_{T}^{i}$ will be also of the same order of magnitude (Eqs. (5] 60) ).

- The presence of the SU(5) partners of $S$ and $T$ induces flavour violating effects in the hadronic sector as well, similarly to what happens in the leptonic sector. Again, the relevant couplings $y_{V}^{i}$, even if in general independent, are expected to be of the same order of $y_{S}^{i}$ and $y_{T}^{i}$.

- The presence of a full $\mathbf{2 4}$ at an intermediate scale between the GUT and the EW scales does not spoil gauge coupling unification if $M_{T} \simeq M_{O} \simeq M_{V} \simeq$ $M_{I}$, as in our case, but affects the gauge couplings running above $M_{I}$. This can have an impact on the SUSY spectrum and, in particular, on the regions of the parameter space which provide a relic density for the LSP within the WMAP bounds, as we are going to discuss in section IV

\section{FLAVOUR VIOLATING PROCESSES}

The presence of the fields in the $\mathbf{2 4}$ modifies the renormalization group running of the parameters of the model, both the superpotential couplings and the SUSY breaking terms. For instance, the renormalization group equations (RGEs) for the scalar masses are now given by:

$$
16 \pi^{2} \frac{d}{d t} m_{\phi}^{2}=\beta_{m_{\phi}^{2}}^{\mathrm{MSSM}, 1}+\beta_{m_{\phi}^{2}}^{\mathbf{2 4 , 1}}
$$


where $\beta_{m_{\phi}^{2}}^{\mathrm{MSSM}, 1}$ is the usual MSSM 1-loop $\beta$-function and $\beta_{m_{\phi}^{2}}^{\mathbf{2 4 , 1}}$ is the new 1-loop contribution given by the new fields in the $\mathbf{2 4}$, with clearly $\beta_{m_{\phi}^{2}}^{\mathbf{2 4 , 1}} \neq 0$ only above the $\mathbf{2 4}$ energy scale.

In particular, the couplings of the seesaw fields, $S$ and $T$, with the lepton doublet will affect the running of the left-handed (LH) slepton masses, generating off-diagonal flavour violating entries, in perfect analogy with what happens in the context of supersymmetric seesaw of type I [18, 22] and type II [11]. In addition, the presence of the $\mathrm{SU}(5)$ partner, $V$, of the seesaw fields will induce an analogous effect for the RH down squarks.

The complete RGEs of the model are given in the Appendix. Let us display here the $\beta$-functions of, respectively, the LH slepton and RH down-squark soft masses, which are the relevant ones for outlining the effects mentioned above:

$$
\begin{aligned}
\left(\beta_{m_{\tilde{L}}^{2}}^{\mathbf{2 4}, 1}\right)_{i j}= & \frac{3}{2}\left(y_{T i}^{*}\left(y_{T}^{T} m_{\tilde{L}}^{2}\right)_{j}+\left(m_{\tilde{L}}^{2} y_{T}^{*}\right)_{i} y_{T j}^{T}+\right. \\
& \left.2 y_{T i}^{*} y_{T j}^{T}\left(m_{H_{u}}^{2}+m_{\tilde{T}}^{2}\right)+2 A_{T i}^{*} A_{T j}^{T}\right)+ \\
& \left(y_{S i}^{*}\left(y_{S}^{T} m_{\tilde{L}}^{2}\right)_{j}+\left(m_{\tilde{L}}^{2} y_{S}^{*}\right)_{i} y_{S j}^{T}+\right. \\
& \left.2 y_{S i}^{*} y_{S j}^{T}\left(m_{H_{u}}^{2}+m_{\tilde{S}}^{2}\right)+2 A_{S i}^{*} A_{S j}^{T}\right),(20) \\
\left(\beta_{m_{\tilde{d}^{c}}^{2}}^{\mathbf{2 4}, 1}\right)_{i j}= & 2\left(y_{V i}^{*}\left(y_{V}^{T} m_{\tilde{d}^{c}}^{2}\right)_{j}+\left(m_{\tilde{d}^{c}}^{2} y_{V}^{*}\right)_{i} y_{V j}^{T}+\right. \\
& \left.2 y_{V i}^{*} y_{V j}^{T}\left(m_{H_{u}}^{2}+m_{\tilde{S}}^{2}\right)+2 A_{V i}^{*} A_{V j}^{T}\right) \cdot(21)
\end{aligned}
$$

Off-diagonal flavour violating entries in the LH slepton and $\mathrm{RH}$ down-squark mass matrices are then generated by RG running from $M_{\mathrm{GUT}}$ down to the seesaw fields mass scales, even starting with universal boundary conditions at $M_{\mathrm{GUT}}, m_{\tilde{L}, \tilde{d}^{c}}^{2}=m_{0}^{2} 1$. From Eqs. (20, 21), we can estimate the flavour violating mass-insertions, $\delta_{i \neq j} \equiv m_{i j}^{2} / \sqrt{m_{i i}^{2} m_{j j}^{2}}$, which parameterize the amount of flavour violation induced by the running. At leadinglog, they read:

$$
\begin{aligned}
\left(\delta_{\mathrm{LL}}^{e}\right)_{i j} & =\frac{1}{8 \pi^{2}} \frac{\left(3 m_{0}^{2}+A_{0}^{2}\right)}{\bar{m}_{\tilde{L}}^{2}} \times \\
& {\left[\frac{3}{2} y_{T}^{i *} y_{T}^{j} \ln \left(\frac{M_{\mathrm{GUT}}}{M_{T}}\right)+y_{S}^{i *} y_{S}^{j} \ln \left(\frac{M_{\mathrm{GUT}}}{M_{S}}\right)\right], } \\
\left(\delta_{\mathrm{RR}}^{d}\right)_{i j} & =\frac{1}{8 \pi^{2}} \frac{\left(3 m_{0}^{2}+A_{0}^{2}\right)}{\bar{m}_{\tilde{d}^{c}}^{2}} 2 y_{V}^{i *} y_{V}^{j} \ln \left(\frac{M_{\mathrm{GUT}}}{M_{V}}\right),
\end{aligned}
$$

where $\bar{m}_{\tilde{L}}^{2}, \bar{m}_{\tilde{d}^{c}}^{2}$ are average slepton and squark squared masses at low energy. Eqs. (22, 23) provide a good estimate of the FV mass-insertions, unless $m_{0}$ is too small. In the case of $m_{0} \simeq 0$, which is indeed possible in the model as we will discuss in the next section, the sfermion masses are generated by the running, but Eqs. (22) 231) are clearly not valid anymore, since sfermion masses are vanishing at $M_{\mathrm{GUT}}$ and possible off-diagonal entries in the mass matrices can be only generated at orders higher than the leading-log.

Keeping that in mind, we can still make use of Eq. (22) to get an idea of the expected amount of LFV. For instance, in the case $M_{S} \simeq M_{T} \simeq 10^{13} \mathrm{GeV}$, we see from the seesaw formula, Eq. (3), that typically $y_{S, T}^{i} \simeq$ $\mathcal{O}\left(10^{-2}\right)$ and therefore assuming $\bar{m}_{\tilde{L}}^{2} \simeq m_{0}^{2}$ and $A_{0} \simeq 0$, Eq. (22) gives roughly:

$$
\left(\delta_{\mathrm{LL}}^{e}\right)_{i j} \simeq \mathcal{O}\left(10^{-4}\right) \quad \text { for } M_{S, T} \simeq 10^{13} \mathrm{GeV},
$$

value which can give sizable effects in the $\mu-e$ transitions only and can already exclude the SUSY parameter space in the light sleptons regime ${ }^{5}$.

We can also estimate the typical ratio of the BRs of different LFV processes. Given that the main source of $\mathrm{LFV}$ is represented by the $\left(\delta_{\mathrm{LL}}^{e}\right)_{i j}$, we have:

$$
\frac{\mathrm{BR}\left(\ell_{i} \rightarrow \ell_{j} \gamma\right)}{\operatorname{BR}\left(\ell_{i} \rightarrow \ell_{j} \nu \bar{\nu}\right)} \propto\left|\left(\delta_{\mathrm{LL}}^{e}\right)_{i j}\right|^{2},
$$

hence the ratio of BRs in the $\tau-\mu$ and $\mu$-e channels can be estimated to be:

$$
R \equiv \frac{\operatorname{BR}(\tau \rightarrow \mu \gamma)}{\operatorname{BR}(\mu \rightarrow e \gamma)} \simeq 0.17 \times \frac{\left|y_{S}^{3 *} y_{S}^{2}+\frac{3}{2} y_{T}^{3 *} y_{T}^{2}\right|^{2}}{\left|y_{S}^{1 *} y_{S}^{2}+\frac{3}{2} y_{T}^{1 *} y_{T}^{2}\right|^{2}} .
$$

Using Eqs. (13, 14), one can check that $4 \lesssim R \lesssim 80$ in the normal hierarchy case for a real parameter $z$, if $U_{e 3} \simeq 0$ and the Majorana phase $\Phi$ vanishes as well. As the value of $U_{e 3}$ increases, one can verify that $R$ diminishes and it becomes $\mathcal{O}(1)$ for $U_{e 3} \simeq 0.2$. Interestingly, as soon as $\operatorname{Im}(z)$ is switched on, $R$ rapidly drops to $\mathcal{O}(1)$ values independently of the value of $U_{e 3}$. As for the role of the phases, they also generically tend to reduce $R$, even if for small non-zero values of $U_{e 3}$ the Dirac phase somehow compensates the $U_{e 3}$ effect, preventing the reduction of the ratio. Moreover, the presence of the phases increases $\left(\delta_{\mathrm{LL}}^{e}\right)_{12}$, from which a bound on $\operatorname{Im}(z)$ can be derived (see later). In the inverted hierarchy case with a real $z, R$ can even diverge, since for certain values of $z\left(\delta_{\mathrm{LL}}^{e}\right)_{12}$ can vanish. However, such cases correspond to set-up of the Yukawas (e.g. $y_{S, T}^{i} \ll y_{T, S}^{i}$ ) which cannot be considered natural in the light of Eqs. (5, 6). Moreover, for $\operatorname{Im}(z) \neq$ 0 , such divergences disappear and $R$ tends to $\mathcal{O}(1)$ values like in the case of normal hierarchy.

Let us briefly make here a comparison with other seesaws, still implemented in a SU(5) context. As already discussed in the previous section, in what respect neutrino masses our model is not different from a model with a type I seesaw with only two RH neutrinos. This statement holds also for LFV, with the only difference given by the fact that in the $2 \mathrm{RHN}$ model the heavy neutrino

\footnotetext{
${ }^{5}$ Cfr. the bounds provided in 23].
} 
masses can be hierarchical, while our model, barring cancellations, predicts $M_{T} \simeq M_{S}$. As a consequence higher values for $R$ can be obtained [20]. In the type I seesaw with three RH neutrinos, due to the larger number of parameters, even more freedom is allowed. On the contrary in the type II seesaw there is a direct relation between high-energy and low-energy neutrino parameters, so that the ratios of the branching ratios of LFV processes can be expressed in terms of neutrino masses and mixing angles. When this is embedded into a SU(5) GUT by adding a $\mathbf{1 5}+\mathbf{1 5}$ representation [11], $R$ varies between 400 and $\mathcal{O}(1)$ with increasing $\theta_{13}$ [24]. Notice that in that model the seesaw fields induce flavour violation in the hadronic sector too, as in the model we are studying in this paper.

Let us now discuss the induced flavour violation in the hadronic sector. From Eq. (23), we see that off-diagonal entries in the $m_{\tilde{d}^{c}}^{2}$ mass matrix are induced by the coupling $\vec{y}_{V}$ of the down-quark SU(2) singlets with the $\mathbf{2 4}$ field $V$. Eqs. (517) tell us that $\vec{y}_{V}$ cannot be unequivocally determined in terms of $\vec{y}_{S}$ and $\vec{y}_{T}$ and, therefore, in terms of neutrino parameters. However, Eqs. (517) also show that $\vec{y}_{V}$ can be naturally expected to be of the same order of magnitude of the seesaw Yukawa couplings, as it clearly follows from the SU(5) embedding of the model. In particular if $\left|\vec{y}_{1,2}\right| \ll\left|\vec{y}_{24}\right|$ in Eqs. (5-7), then $\vec{y}_{V} \simeq \vec{y}_{T}$. In our numerical analysis, we are going to make use of this last assumption ${ }^{6}$. Anyway, with a free choice of $\vec{y}_{1,2}$ and barring cancellations, we would still get:

$$
\left(\delta_{\mathrm{RR}}^{d}\right)_{i j} \simeq\left(\delta_{\mathrm{LL}}^{e}\right)_{i j} \frac{\bar{m}_{\tilde{L}}^{2}}{\bar{m}_{\tilde{d}^{c}}^{2}} .
$$

Thus, comparing this expression with Eq. (24), we find that the typical order of magnitude of the hadronic mass insertion is:

$$
\left(\delta_{\mathrm{RR}}^{d}\right)_{i j} \simeq \mathcal{O}\left(10^{-5} \div 10^{-4}\right) \text { for } M_{V} \simeq 10^{13} \mathrm{GeV},
$$

with $\left(\delta_{\mathrm{RR}}^{d}\right)_{i j}$ becoming maximal for $m_{0} \gg M_{1 / 2}$, when $\bar{m}_{\tilde{d}^{c}}^{2} \simeq \bar{m}_{\tilde{L}}^{2} \simeq m_{0}^{2}$. Moreover, one has to take into account that, like in the MSSM, the RGE for $m_{\tilde{Q}}^{2}$ generates also small $\left(\delta_{\mathrm{LL}}^{d}\right)_{i j}$ proportional to CKM elements, $\left(\delta_{\mathrm{LL}}^{d}\right)_{i j} \propto V_{t i}^{*} V_{t j}$. Taking into account this further effect, the most stringent bounds, which come from the Kaon system, are $\left|\left(\delta_{\mathrm{RR}}^{d}\right)_{12}\right| \simeq \mathcal{O}\left(10^{-3}\right)[25]$. Comparing this value with Eq. (28), we see that the model does not typically predict large deviations from the constrained MSSM (CMSSM) predictions in the hadronic sector and therefore it is safe from hadronic FCNC constraints. However, Eq. (28) provides a quite rough estimate and it is worth to study in more detail some hadronic observables, for

\footnotetext{
${ }^{6}$ Another possible approach to improve the predictivity of the model in the hadronic sector is considering the renormalizable version of the model discussed in [6], where $\vec{y}_{V}$ can be written as a combination of $\vec{y}_{T}$ and $\vec{y}_{S}$. Apart from this point, that model gives the same phenomenology discussed here.
}

which experiments have recently showed possible tensions with the SM (and the CMSSM) predictions. In the next section, in particular, we are going to comment about the impact of the new flavour mixing sources of the model on the Kaon CP-violating parameter $\epsilon_{K}$ and on the time-dependent CP asymmetry, $S_{\psi \phi}$, in the decay $B_{s} \rightarrow J / \psi \phi$.

\section{RESULTS}

As mentioned above, in order to outline the effects induced by the RG running between the GUT scale and the mass scale of the $\mathbf{2 4}$ fields, we consider universal boundary conditions, namely: a universal scalar mass $m_{0}$, a common gaugino mass $M_{1 / 2}$ and trilinear terms $A_{f}=A_{0} Y_{f}$.

In order to compute the SUSY spectrum, we numerically solve the full 1-loop RGEs of the model (see the Appendix) down to the seesaw scale, $M_{S}=M_{T} \equiv M_{I}$, at which the $\mathbf{2 4}$ fields decouple. Then, we run the MSSM RGEs down to the SUSY scale $m_{\mathrm{SUSY}} \equiv \sqrt{m_{\tilde{t}_{1}} m_{\tilde{t}_{2}}}$. For each point of the parameter space, we impose the following requirements: (i) successful EWSB and absence of tachyonic particles; (ii) limits on SUSY masses from direct searches; (iii) neutral LSP. Then, we compute the leptonic processes by means of a full calculation in the mass eigenstate basis [26], the hadronic processes by means of the mass-insertion approximation formulae in [27], the LSP relic density using DarkSUSY [28] and the $\operatorname{BR}\left(B \rightarrow X_{s} \gamma\right)$ using SusyBSG [29]. We require that the resulting $\mathrm{BR}\left(B \rightarrow X_{s} \gamma\right)$ do not deviate from the experimental value [30] in more than $3 \sigma$.

Let us first try to extract information about the seesaw scale and the other seesaw parameters, focusing on the stringent bounds LFV can impose on them. In order to do that, we can start varying all the parameters in large ranges, but we clearly need a criterion for defining the SUSY spectrum we want to concentrate on (all effects would be negligibly small, if we considered slepton masses of several TeV). Therefore, we will mostly concentrate on parameter regions giving sizable SUSY contributions to the anomalous magnetic moment of the muon, $(g-2)_{\mu}$, and, later on, also on regions which provide a dark matter relic density within the WMAP constraints.

For simplicity, in the numerical analysis we have neglected possible $\mathcal{O}(1)$ mass-splittings among the fields in the 24, inducing threshold corrections to the gauge coupling running, which would modify the MSSM gauge coupling unification. In particular, the 1-loop prediction for the value of the strong coupling at $M_{Z}$ would become:

$$
\frac{1}{\alpha_{3}\left(M_{Z}\right)}-\frac{1}{\alpha_{3}^{0}\left(M_{Z}\right)}=\frac{3}{2 \pi}\left[\ln \frac{M_{T}}{M_{O}}+\frac{1}{7} \ln \frac{M_{T}}{M_{V}}\right],
$$

where $\alpha_{3}^{0}\left(M_{Z}\right)$ is the 1-loop MSSM prediction. According to the above formula, the consistency with the measured value for $\alpha_{3}\left(M_{Z}\right)$ could be slightly worsened or improved. We notice that such modification of the running of the 

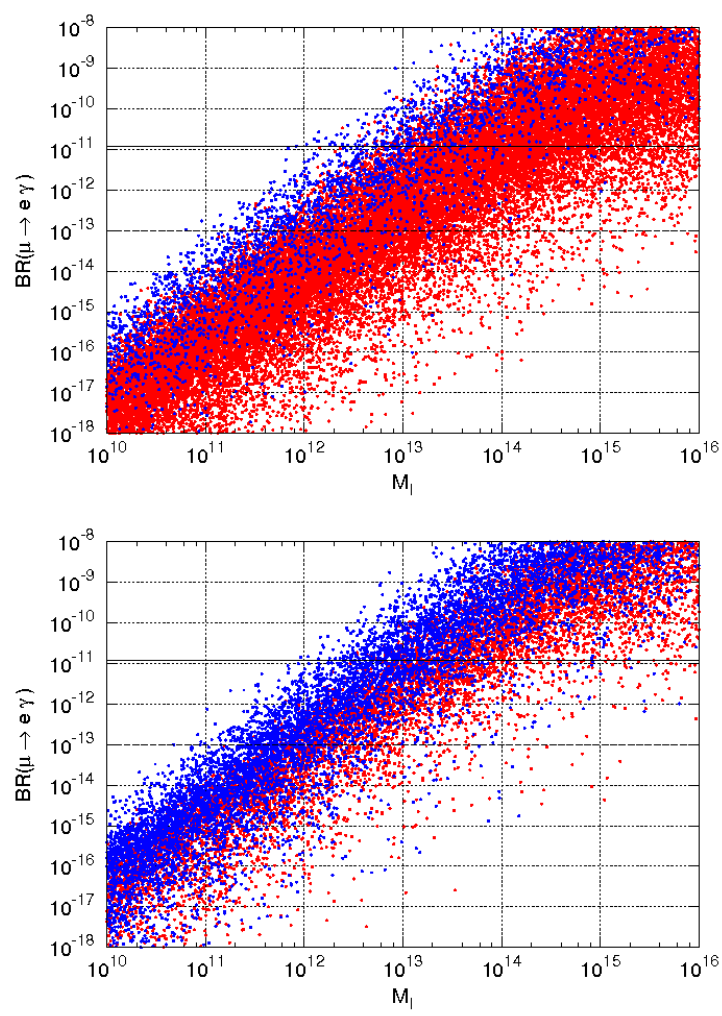

FIG. 1: $\mathrm{BR}(\mu \rightarrow e \gamma)$ vs. the seesaw scale $M_{I}$ (in $\mathrm{GeV}$ ) for $\tan \beta=10$ (top) and $\tan \beta=40$ (bottom) and a wide scan of the parameters (see the text for details). Blue points provide $(g-2)_{\mu}^{\mathrm{SUSY}} / 2>10^{-9}$.

gauge couplings would have anyway a negligible impact on the running of the other parameters. Moreover, the possible thresholds would affect the running of the soft masses, entering in the expressions of the flavour violating parameters, Eqs. (22] 23), only logarithmically, so that they would have a small effect on the observables we are going to study.

\section{A. Lepton Flavour Violation}

In Fig. 1. we plot $\operatorname{BR}(\mu \rightarrow e \gamma)$ as a function of the seesaw scale $M_{S}=M_{T} \equiv M_{I}$, in the case of normal neutrino hierarchy ${ }^{7}$, for the following choice of the parameters: $\tan \beta=10$ (top panel) and $\tan \beta=40$ (bottom panel), $0<m_{0}<1 \mathrm{TeV}, 0<M_{1 / 2}<1.5 \mathrm{TeV}$, $\left|A_{0}\right| \leq 3 m_{0}$. The neutrino parameters were also varied in the following ranges: $10^{10} \mathrm{GeV} \leq M_{I} \leq 10^{16} \mathrm{GeV}$, $10^{-3} \leq\left|U_{e 3}\right| \leq 0.2,0 \leq z<2 \pi$. We took the parameter

\footnotetext{
7 We checked that inverted hierarchical neutrinos do not provide significantly different predictions with respect to the normal hierarchy case. We will thus concentrate on normal hierarchy from now on.
}

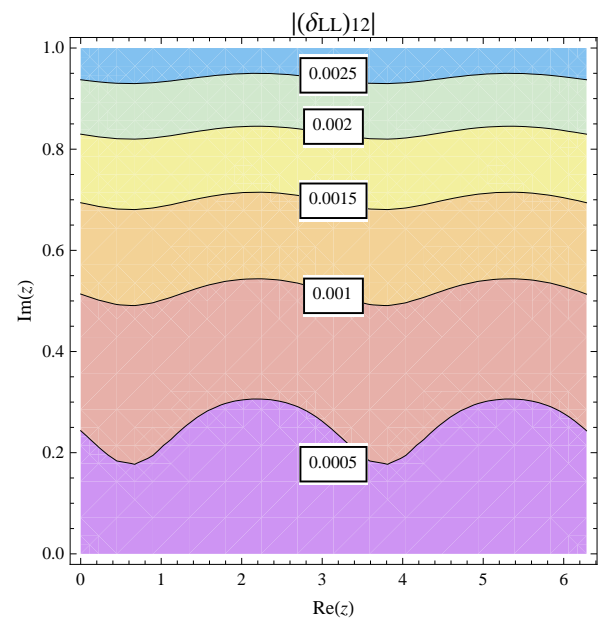

FIG. 2: Contour plot for the LFV parameter $\left|\left(\delta_{\mathrm{LL}}^{e}\right)_{12}\right|$ in the $\operatorname{Re}(z)-\operatorname{Im}(z)$ plane, for $m_{0}=M_{1 / 2}, A_{0}=0, M_{I}=10^{13} \mathrm{GeV}$ and $U_{e 3}=0, \Phi=0$.

$z$ real, since the only effect of its imaginary part is to raise the seesaw Yukawas and so the $\mu \rightarrow e \gamma$ rate, as we commented in Sec. III. However, $\operatorname{BR}(\mu \rightarrow e \gamma)$ itself provides very stringent bounds on $\operatorname{Im}(z)$, as we will comment below. We have also checked that all couplings remain perturbative up to the GUT scale. The blue (black) points give $a_{\mu}^{\mathrm{SUSY}} \equiv(g-2)_{\mu}^{\mathrm{SUSY}} / 2>10^{-9}$, so lowering the tension between theoretical prediction and experiments below the $2 \sigma$ level.

For $\tan \beta=10$ (upper panel of Fig. 1), we see, besides the dependence $\operatorname{BR}(\mu \rightarrow e \gamma) \sim M_{I}^{2}$, that the current experimental limit $\mathrm{BR}(\mu \rightarrow e \gamma)<1.2 \times 10^{-11}$ 31], constrains the seesaw scale to be $M_{I} \lesssim 10^{13} \div 10^{14} \mathrm{GeV}$ for the points favored by $(g-2)_{\mu}$, even if there are few points, for which the parameters conspire in lowering $\operatorname{BR}(\mu \rightarrow e \gamma)$, that can evade such bound. Even if $\operatorname{BR}(\mu \rightarrow e \gamma)$ is enhanced by increasing $\tan \beta$, we find the above limit on the seesaw scale also for $\tan \beta=40$ (lower panel of Fig. 1), since $a_{\mu}^{\mathrm{SUSY}}$ is increased by $\tan \beta$ as well. In both cases, the MEG experiment [32], whose expected sensitivity is $\operatorname{BR}(\mu \rightarrow e \gamma) \simeq 10^{-13}$, will be able to test soon the region of the parameter space favored by $(g-2)_{\mu}$ down to $M_{I} \simeq 10^{12} \div 10^{13} \mathrm{GeV}$.

Let us now show how the present bound on $\operatorname{BR}(\mu \rightarrow$ $e \gamma$ ) can severely constrain the parameter $z$. We have already argued above that $\operatorname{Im}(z)$ cannot be too large, without having unnatural cancellations in the neutrino mass matrix $m_{\nu}$ between the singlet and the triplet terms. Besides that, LFV bounds can directly constrain $\operatorname{Im}(z)$, since the seesaw Yukawas simply grow by increasing it. For convenience, let us express the $\operatorname{BR}(\mu \rightarrow e \gamma)$ constraints in terms of bounds on the mass insertion $\left(\delta_{\mathrm{LL}}^{e}\right)_{12}$. From the same scan of the parameters presented above, we find that satisfying the present limit on $\mu \rightarrow e \gamma$ requires:

$$
\left|\left(\delta_{\mathrm{LL}}^{e}\right)_{12}\right|<(5 \div 6) \times 10^{-4}
$$




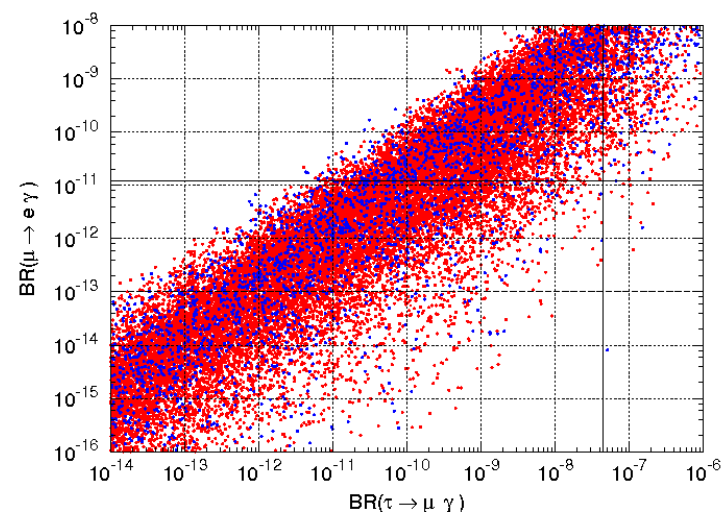

FIG. 3: $\operatorname{BR}(\mu \rightarrow e \gamma)$ vs. $\operatorname{BR}(\tau \rightarrow \mu \gamma)$, for $\tan \beta=10$ and the same scan of the parameters of Fig. 1]

for the points lying in the blue (black) region. In Fig. 2 , we show contours for $\left|\left(\delta_{\mathrm{LL}}^{e}\right)_{12}\right|$ in the $\operatorname{Re}(z)-\operatorname{Im}(z)$ plane, for $m_{0}=M_{1 / 2}, A_{0}=0, M_{I}=10^{13} \mathrm{GeV}$ and $U_{e 3}=0$, $\Phi=0$. We see that, indeed, $\left|\left(\delta_{\mathrm{LL}}^{e}\right)_{12}\right|$ grows very fast with $\operatorname{Im}(z)$. As a consequence, the bound of Eq. (30) constrains $\operatorname{Im}(z)$ to values $\lesssim 0.3$ for the seesaw scale at $10^{13} \mathrm{GeV}$.

Let us finally consider LFV in the $\mu-\tau$ sector as well. In Fig. 3, we plot $\operatorname{BR}(\mu \rightarrow e \gamma)$ vs. $\operatorname{BR}(\tau \rightarrow \mu \gamma)$, for $\tan \beta=$ 10 and the same variation of the other parameters of Fig. 1. We see that in this model, the strong bound on flavour transition in the $\mu$-e sector already challenges future $\tau \rightarrow \mu \gamma$ experiments quite strongly. In fact, the bulk of the points, for which $\operatorname{BR}(\mu \rightarrow e \gamma)$ is less than the present bound, gives $\operatorname{BR}(\tau \rightarrow \mu \gamma) \lesssim 10^{-9}$, which is indeed below the expected sensitivity of the proposed Super Flavour Factory [33]. This is consistent with the estimate for $R=\operatorname{BR}(\tau \rightarrow \mu \gamma) / \operatorname{BR}(\mu \rightarrow e \gamma) \lesssim 100$, we provided in the previous section. However, we see that there are some points for which parameters conspire to raise the value of $\operatorname{BR}(\tau \rightarrow \mu \gamma)$ at the level of $10^{-8}$, i.e. in the reach of the SuperB factory at KEK 34]. Nevertheless, a positive signal for $\operatorname{BR}(\tau \rightarrow \mu \gamma)$ would anyway disfavor the scenario under study.

\section{B. Hadronic observables}

In this section, we are going to discuss the effects of the new source of flavour mixing in the down squark sector, Eq. (23), induced by the running between the GUT and the seesaw scales. In particular, it is interesting to check if this is able to account for a large phase in the $B_{s}$ mixing, as suggested by the Tevatron experiments CDF [35] and D0 [36, 37]. Moreover, a positive new physics contribution to $\epsilon_{K}$ (around the $24 \%$ of the SM contribution) [38] is one of the possible ways for accommodating a recently reported tension among different observables used to fit the unitarity triangle (see also [25, 39]).

As pointed out in section [II] hadronic flavour mixing cannot be directly related to the leptonic one. Never-
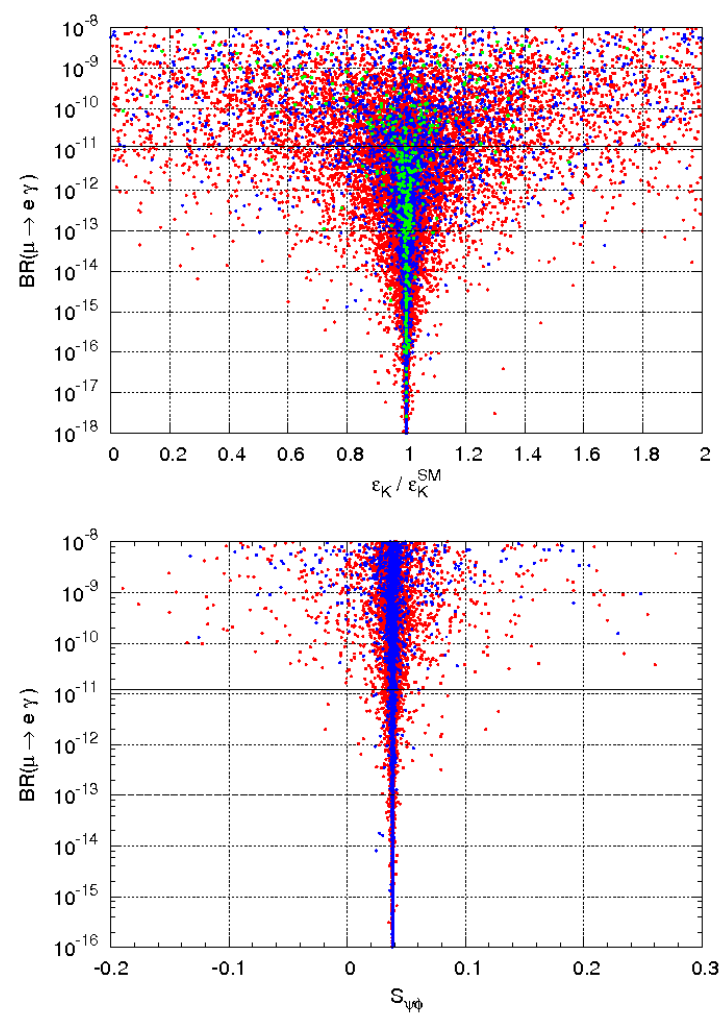

FIG. 4: Top: $\operatorname{BR}(\mu \rightarrow e \gamma)$ vs. $\epsilon_{K} / \epsilon_{K}^{\mathrm{SM}}$ for $10 \leq \tan \beta \leq$ 40 and the same scan of parameters as in Fig. 1] Bottom: $\operatorname{BR}(\mu \rightarrow e \gamma)$ vs. $S_{\psi \phi}$.

theless, the off-diagonal entries of $m_{\tilde{d}^{c}}^{2}$ are naturally of the same order of magnitude as the leptonic ones. For definitiveness, we take $\vec{y}_{V}=\vec{y}_{T}$ as input for the RGEs at the GUT scale, then we vary the phases of the resulting low-energy mass insertions $\left(\delta_{\mathrm{RR}}^{d}\right)_{i j}$ between 0 and $2 \pi$.

Let us start to consider the possible effect of the generated $\left(\delta_{\mathrm{RR}}^{d}\right)_{12}$ on the SUSY contribution to $\epsilon_{K}$. In the top panel of Fig. 4 , we plot $\mathrm{BR}(\mu \rightarrow e \gamma)$ vs. $\epsilon_{K} / \epsilon_{K}^{\mathrm{SM}}$ for $10 \leq \tan \beta \leq 40$ and the same variation of the parameters as in Fig. 1. As for the previous plots, the blue (black) points provide a sizable SUSY contribution to $(g-2)_{\mu}$, while the green (light grey) points give a neutralino relic density not larger than the cold dark matter relic density measured by WMAP (see the next section for details). We can see that the present bound on $\operatorname{BR}(\mu \rightarrow e \gamma$ ) still allows for a sizable (up to $20 \div 30 \%$ of $\left.\epsilon_{K}^{\mathrm{SM}}\right)$ positive contribution to $\epsilon_{K}$. Furthermore we have $\operatorname{BR}(\mu \rightarrow e \gamma)>10^{-13}$ (so within the sensitivity of MEG) for most of the points which provide such a solution to the $\epsilon_{K}$ tension, which would be therefore strongly disfavored by a negative result of MEG. Let us notice, however, that the parameter space points favored by WMAP cannot provide the desired increase of $\epsilon_{K}$. The reason is that these points are mainly concentrated in the coannihilation region where $M_{1 / 2}>m_{0}$ or even $M_{1 / 2} \gg m_{0}$, as we are going to discuss in the next section, so that the flavour violating $\left(\delta_{\mathrm{RR}}^{d}\right)_{i j}$ result suppressed by large 
squark masses.

In the bottom panel of Fig. 4. we plot $S_{\psi \phi}$ for the same scan of the parameters. As we can see, the predicted value do not deviate too much from the small SM prediction $S_{\psi \phi}^{\mathrm{SM}} \simeq 0.036$. The reason is that, even if the phase of $\left(\delta_{\mathrm{RR}}^{d}\right)_{23}$ can be large, $\left|\left(\delta_{\mathrm{RR}}^{d}\right)_{23}\right|$ is numerically too small (cfr. for instance the estimate in Eq. (28)) to provide a sizable $\mathrm{CP}$ violation in the $B_{s}$ mixing and thus accounting for the di-muon anomaly reported in [37]. If such new physics effects in $B_{s}$ mixing will be confirmed, the minimal version of the model we are discussing here should be extended to include further sources of flavour violation in the hadronic sector.

\section{Neutralino relic density}

The presence of intermediate scale fields, which are charged under the SM gauge group, has a possible impact on the supersymmetric spectrum and, thus, on the parameter space regions, for which the relic density of the LSP (in our case a bino-like lightest neutralino as in the CMSSM) results to be within the WMAP bounds [40]. In this section, we are going to focus on the socalled $\tilde{\tau}$ coannihilation region [41], since focus point 42] and A-funnel [43] are not expected to be qualitatively different with respect to the CMSSM (even if they can be quantitatively modified, even significantly, within this model).

The effect we are going to discuss can be again traced back to the modification of the RG running of the parameters. In this case, however, this is not due to the new Yukawa interactions (since flavour bounds do not allow the couplings to be too large), but it is an effect of the modification of the running of the gauge couplings (and the gaugino masses) above the scale of the $\mathbf{2 4}$. In fact, even if the fields in the $\mathbf{2 4}$ do not spoil (at least at 1-loop) the successful gauge coupling unification of the MSSM, the running gets "stronger": above $M_{T} \simeq M_{V} \simeq M_{O}$ the 1-loop $\beta$-function coefficients gets indeed modified as follows:

$$
b_{i}=b_{i}^{\mathrm{MSSM}}+b_{i}^{24}=(33 / 5,1,-3)+(5,5,5),
$$

and the running of the gauge couplings is considerably deflected. As a consequence, even if the couplings unify at the usual MSSM GUT scale, $M_{\mathrm{GUT}} \sim 10^{16} \mathrm{GeV}$, the value of the unified coupling $\alpha_{U}$ gets larger than in the CMSSM.

Clearly, an analogous effect happens to the gaugino masses, so that they reach values at $M_{I}$, which can be considerably smaller than the unified value $M_{1 / 2}$. This could be thought as a simple rescaling of $M_{1 / 2}$ (since clearly the low-energy gaugino masses will be the same as in the CMSSM with a lower value of $M_{1 / 2}$ ), if it did not affect the scalar masses as well. In fact, with the same values of the gaugino masses at low energy, the scalar mass will feel a stronger gauge contribution to the

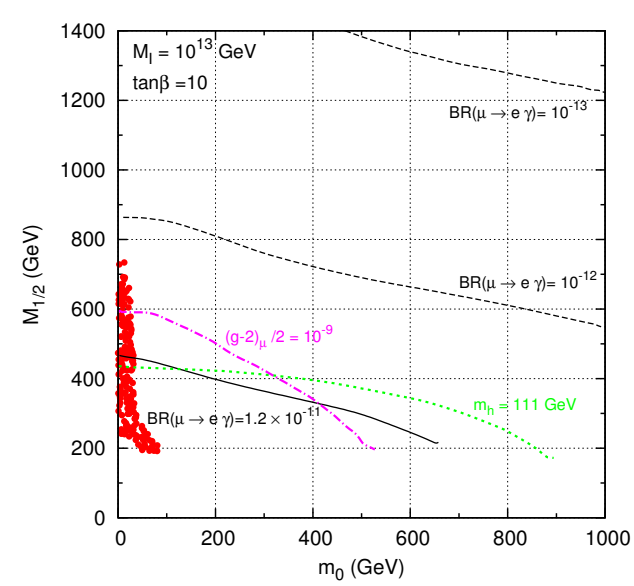

FIG. 5: Coannihilation region and $\mathrm{BR}(\mu \rightarrow e \gamma)$ contours in the $\left(m_{0}, M_{1 / 2}\right)$ plane, for $\tan \beta=10, M_{I}=10^{13} \mathrm{GeV}$.

running, through the gauge terms in the RGEs, $\sim \alpha_{i} M_{i}^{2}$, which are larger than in the CMSSM between the GUT scale and $M_{I}$. The consequence in the low-energy SUSY spectrum is that the scalar masses will result relatively larger, with respect to the gaugino masses, than in the MSSM.

Qualitatively, the above described effect is clearly common to all models that have fields charged under the SM gauge group at some intermediate scale, and it was, for instance, observed in the context of an $S O(10)$ type-II seesaw model in 44] and in a multi-scale flavour model in [45].

Coming back to DM, having relatively heavier scalars could destabilize the ordinary regions of the parameter space that provide a neutralino relic density within the WMAP bounds and for which quite precise relations among parameters are usually required. An example is the $\tilde{\tau}$ coannihilation region, where the correct relic density is achieved thanks to an efficient $\tilde{\tau}-\tilde{\chi}_{1}^{0}$ coannihilation, which requires $m_{\tilde{\tau}_{1}} \simeq m_{\tilde{\chi}_{1}^{0}}$. As we are going to see, such region is strongly modified in our case, as an effect of the relatively heavier $\tilde{\tau}_{1}$ resulting from the strong gauge running below the GUT scale.

What can happen to the coannihilation region is depicted in Fig. 5], where we show the $m_{0}-M_{1 / 2}$ plane for $M_{I}=10^{13} \mathrm{GeV}, \tan \beta=10, A_{0}=0$. The neutrino parameters (not relevant for $\mathrm{DM}$ ) were taken to be $U_{e 3}=0$, $z=1$. The region marked with red (grey) points gives $\Omega_{\mathrm{DM}} h^{2} \leq 0.13$. We can see that the CMSSM region where $m_{\tilde{\tau}_{1}}<m_{\tilde{\chi}_{1}^{0}}$, along which usually the coannihilation strip runs, has disappeared as a consequence of the effect described above ${ }^{8}$. Coannihilation is still pos-

\footnotetext{
8 This opens up the possibility of having $m_{0}=0$, i.e. vanishing scalar masses at high-energy (then generated through the running driven by the gaugino masses), such as in [44, 45]. This possibility has been recently addressed in [46].
} 


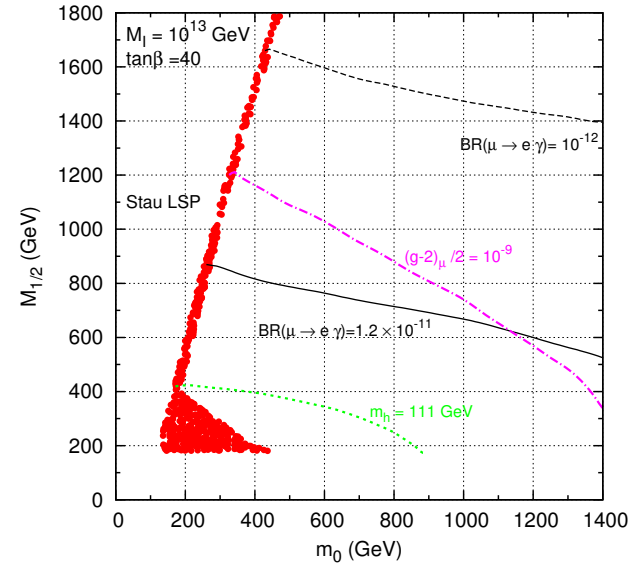

FIG. 6: Coannihilation region and $\operatorname{BR}(\mu \rightarrow e \gamma)$ contours in the $\left(m_{0}, M_{1 / 2}\right)$ plane, for $\tan \beta=40, M_{I}=10^{13} \mathrm{GeV}$.

sible, since very low values of $m_{0}$ still gives $m_{\tilde{\tau}_{1}} \simeq m_{\tilde{\chi}_{1}^{0}}$, but, interestingly, such region is bounded from above: this means that this particular set-up of the parameters predicts an upper bound on the DM mass, in this case $m_{\tilde{\chi}_{1}^{0}} \lesssim 240 \mathrm{GeV}$, as we can see from the figure taking into account that, for $M_{I}=10^{13} \mathrm{GeV}$, the bino mass is approximately $M_{1} \simeq 0.31 M_{1 / 2}$. A similar effect, providing an upper bound on $m_{\tilde{\chi}_{1}^{0}}$, was found in [47], again as a consequence of the modification of the gauge contribution to the running of the scalar masses ${ }^{9}$ (in that case an additional $\mathrm{SU}(5)$ running of the parameters above the GUT scale was taken into account).

In Fig. [5, we have also plotted contours for $\mathrm{BR}(\mu \rightarrow$ $e \gamma$ ), the LEP limit on the Higgs mass (taking into account $3 \mathrm{GeV}$ of theoretical error), as well as the region which provides $(g-2)_{\mu}^{\mathrm{SUSY}} / 2>10^{-9}$ (below the magenta dotdashed line). We can see that the DM region is already partially excluded by the present limits on $\mu \rightarrow e \gamma$ and on the Higgs mass. The rest of the coannihilation region, which is, at least in part, consistent with a sizable $(g-$ $2)^{\text {SUSY }}$, will be fully tested very soon by MEG, since it gives $\operatorname{BR}(\mu \rightarrow e \gamma)>10^{-12}$. The prediction for the $\mu \rightarrow e \gamma$ rate clearly depends on the parameter $z$, which we have here taken $z=1$. Nevertheless, we checked that, varying the value of $z$, still $\operatorname{BR}(\mu \rightarrow e \gamma)$ is predicted in the reach of the MEG experiment (i.e. $\gtrsim 10^{-13}$ ) in the coannihilation region, apart from few points where the combination of parameters happens to suppress the rate.

Finally, it is important to stress how the effect described above and its possible impact on the coannihilation region are sensitive to variations of the parameters, especially $M_{I}$ and $\tan \beta$. The effect would be clearly decreased, and would eventually disappear, by increasing $M_{I}$, i.e. decreasing the length of the running and thus

\footnotetext{
${ }^{9}$ See also 48 50].
}

the value of $\alpha_{U}$, and vice-versa would become stronger for lower values of $M_{I}$. A larger value of $\tan \beta$ would contribute as usual to decrease the $\tilde{\tau}_{1}$ mass (by increasing the negative contributions $\propto y_{\tau}^{2}$ in the running and also the L-R mixing term in the $\tilde{\tau}$ mass matrix). This can be seen in Fig. 6, where the $\tan \beta=40$ case is shown. The parameter space is now qualitatively similar to the CMSSM case: the region where $\tilde{\tau}_{1}$ is the LSP has reappeared and the coannihilation region is a strip along it. Notice the presence for low values of $m_{0}$ and $M_{1 / 2}$ of a sizable "bulk" region (which is smaller but still present also for $\tan \beta=10)$. This region is, however, already excluded by several constraints, including the experimental limit on $\operatorname{BR}(\mu \rightarrow e \gamma)$.

\section{DISCUSSION AND CONCLUSIONS}

In this paper we have considered a SUSY SU(5) model where neutrino masses are obtained via a mixed type I+III seesaw mechanism and we have studied its phenomenology assuming universal soft masses at the GUT scale. The main characteristic of the model is the presence of one massless neutrino. Then the high-energy seesaw parameters are less than in the three massive neutrinos case and therefore a higher degree of predictability is present. Moreover, the model represents a very economical way of accounting for neutrino masses in a GUT context, since the addition of just one chiral superfield in the $\mathrm{SU}(5)$ adjoint representation is considered.

Besides discussing the model and in particular the mechanism through which we obtain two neutrino masses, we have analyzed the following features:

- $\mu \rightarrow e \gamma$ and other LFV processes;

- possible contributions to hadronic observables;

- neutralino relic density.

We have shown that we can have sizable contribution to the $\mu \rightarrow e \gamma$ rate, such that the current experimental limit constrains the seesaw scale to be $M_{I} \lesssim$ $10^{13} \div 10^{14} \mathrm{GeV}$, while MEG will be able to test the model down to scales of $M_{I} \lesssim 10^{12} \div 10^{13} \mathrm{GeV}$. We have also shown that the bounds on $\operatorname{BR}(\mu \rightarrow e \gamma)$ put strong constraints on $\operatorname{BR}(\tau \rightarrow \mu \gamma)$, making very unlikely to observe it in future experiments. Otherwise, a positive signal for the $\tau \rightarrow \mu \gamma$ decay would disfavor this model.

From the bound on $\operatorname{BR}(\mu \rightarrow e \gamma)$, we have been able to put an upper bound on $\operatorname{Im}(z) \lesssim 0.3$, for the seesaw scale $M_{I}=10^{13} \mathrm{GeV}$. Of course this bound is $M_{I}$-dependent, since a reduction in the scale would imply a decrease of the size of Yukawas and then larger values for $\operatorname{Im}(z)$ would be allowed. However, as discussed in Sect. [II, values of $\operatorname{Im}(z)$ larger than 1 are unnatural since cancellations in the neutrino sector would be needed.

The contribution in the hadronic sector is given by the coupling of the down-quark singlets with the new fields 
$V$. Even if this cannot be directly related to the neutrino parameters, an order of magnitude estimate can be performed. We have shown that in this model the present bound on $\operatorname{BR}(\mu \rightarrow e \gamma)$ still allows for a sizable (up to $20 \div 30 \%$ of $\epsilon_{K}^{\mathrm{SM}}$ ) positive contribution to $\epsilon_{K}$, which would help in accommodating a recently reported tension among different observables used to fit the unitarity triangle. On the other side, $\mathrm{CP}$ violation in the $B_{s}$ mixing turns out to be too small to be able to account for the di-muon anomaly reported by the D0 collaboration.

As for the neutralino relic density, we have focussed on the so-called $\tilde{\tau}$ coannihilation region. We have shown that the CMSSM region where $m_{\tilde{\tau}_{1}}<m_{\tilde{\chi}_{1}^{0}}$, along which usually the coannihilation strip runs, can disappear, so that the coannihilation region gets distorted. Interestingly, such region is bounded from above, which means that an upper bound on the DM mass can be derived: for the particular set-up of the parameters considered here we got $m_{\tilde{\chi}_{1}^{0}} \lesssim 240 \mathrm{GeV}$. Moreover, the possibility of having $m_{0}=0$ (with efficient coannihilation as well) as high-energy boundary condition is now open.

In this paper we have not addressed other issues such as proton decay and leptogenesis. As for proton decay, this model does not improve the situation with respect to the standard case, so one has to rely on standard mechanisms to suppress the proton decay rate. For instance, in the context of the missing partner mechanism [51] for solving the doublet-triplet splitting problem, it is possible to build models in which the proton decay rate is sufficiently suppressed [52]. An extended Higgs sector is required in that case. In principle, our model could be embedded in such extended SU(5) framework. For a review about proton stability, including also a section about SUSY SU(5) models, where further possibilities for suppressing the proton decay are discussed, we refer to 53 .
For what concerns leptogenesis, we argue that it can be realized in this model through the decay of the triplet or the singlet or both. However, since their exact masses are not determined from phenomenological constraints (contrary to the non-SUSY case addressed in Ref. [54]), it is not clear who is the responsible for leptogenesis: actually a combined action of the two could be possible, since their masses are of the same order of magnitude. To derive a definite conclusion as well as bounds on the parameters, a dedicated study would then be needed, which is beyond the scope of this work.

Acknowledgments: We are grateful to Michele Frigerio and Paride Paradisi for useful discussions. We also acknowledge the hospitality and partial support of the Galileo Galilei Institute for Theoretical Physics (GGI), Firenze, where part of this work was carried out.

\section{Appendix A: Renormalization group equations}

We present here the complete RGEs for this model. In the case of the MSSM parameters, we only explicitly write the new contributions to the 1-loop $\beta$-functions, according to the definition:

$$
16 \pi^{2} \frac{d}{d t} X=\beta_{X}^{\mathrm{MSSM}, 1}+\beta_{X}^{\mathbf{2 4 , 1}},
$$

where $X$ can represent either Yukawa couplings, A-terms or soft masses. Clearly, all $\beta_{X}^{\mathbf{2 4 , 1}}$ vanishes below the scale of the involved 24 fields. The corresponding $\beta_{X}^{\mathrm{MSSM}, 1}$ can be found, for instance, in 55]. For the new non-MSSM parameters, we provide the complete 1 -loop $\beta$-functions, still denoted as $\beta_{X}^{\mathbf{2 4 , 1}}$.

We first write the $\beta_{X}^{\mathbf{2 4}, 1}$ for the Yukawa couplings:

$$
\begin{aligned}
\left(\beta_{Y_{u}}^{\mathbf{2 4}, 1}\right)_{i j} & =\left(\frac{3}{2}\left|\vec{y}_{T}\right|^{2}+\left|\vec{y}_{S}\right|^{2}+3\left|\vec{y}_{V}\right|^{2}\right)\left(Y_{u}\right)_{i j} \\
\left(\beta_{Y_{d}}^{\mathbf{2 4}, 1}\right)_{i j} & =2 y_{V i}\left(\vec{y}_{V}^{\dagger} Y_{d}\right)_{j} \\
\left(\beta_{Y_{e}}^{\mathbf{2 4}, 1}\right)_{i j} & =\frac{3}{2}\left(Y_{e} \vec{y}_{T}^{*}\right)_{i} y_{T j}^{T}+\left(Y_{e} \vec{y}_{S}^{*}\right)_{i} y_{S j}^{T} \\
\left(\beta_{y_{S}}^{\mathbf{2 4}, 1}\right)_{i} & =\left(-\frac{3}{5} g_{1}^{2}-3 g_{2}^{2}+3 \operatorname{Tr}\left(Y_{u} Y_{u}^{\dagger}\right)+4\left|\overrightarrow{y_{S}}\right|^{2}+\frac{3}{2}\left|\overrightarrow{y_{T}}\right|^{2}+3\left|\overrightarrow{y_{V}}\right|^{2}\right) y_{S i}+\frac{3}{2}\left(\overrightarrow{y_{T}^{*}} \cdot \overrightarrow{y_{S}}\right) y_{T i}+\left(Y_{e}^{T} Y_{e}^{*} \overrightarrow{y_{S}}\right)_{i} \\
\left(\beta_{y_{T}}^{\mathbf{2 4}, 1}\right)_{i} & =\left(-\frac{3}{5} g_{1}^{2}-7 g_{2}^{2}+3 \operatorname{Tr}\left(Y_{u} Y_{u}^{\dagger}\right)+\left|\overrightarrow{y_{S}}\right|^{2}+4\left|\overrightarrow{y_{T}}\right|^{2}+3\left|\overrightarrow{y_{V}}\right|^{2}\right) y_{T i}+\left(\overrightarrow{y_{S}^{*}} \cdot \overrightarrow{y_{T}}\right) y_{S i}+\left(Y_{e}^{t} Y_{e}^{*} \vec{y}_{T}\right)_{i} \\
\left(\beta_{y_{V}}^{\mathbf{2 4}, 1}\right)_{i} & =\left(-\frac{19}{15} g_{1}^{2}-3 g_{2}^{2}-\frac{16}{3} g_{3}^{2}+3 \operatorname{Tr}\left(Y_{u} Y_{u}^{\dagger}\right)+\left|\overrightarrow{y_{S}}\right|^{2}+\frac{3}{2}\left|\overrightarrow{y_{T}}\right|^{2}+6\left|\overrightarrow{y_{V}}\right|^{2}\right) y_{V i}+2\left(Y_{d} Y_{d}^{\dagger} \overrightarrow{y_{V}}\right)_{i}
\end{aligned}
$$


The 1-loop $\beta$-functions for the soft scalar masses read:

$$
\begin{aligned}
\left(\beta_{m_{\tilde{L}}^{2}}^{\mathbf{2 4}, 1}\right)_{i j}= & \frac{3}{2}\left(y_{T i}^{*}\left(\vec{y}_{T}^{T} m_{\tilde{L}}^{2}\right)_{j}+\left(m_{\tilde{L}}^{2} \vec{y}_{T}^{*}\right)_{i} y_{T j}^{T}+2 y_{T i}^{*} y_{T j}^{T}\left(m_{H_{u}}^{2}+m_{\tilde{T}}^{2}\right)+2 A_{T i}^{*} A_{T j}^{T}\right)+ \\
& \left(y_{S i}^{*}\left(\vec{y}_{S}^{T} m_{\tilde{L}}^{2}\right)_{j}+\left(m_{\tilde{L}}^{2} \vec{y}_{S}^{*}\right)_{i} y_{S j}^{T}+2 y_{S i}^{*} y_{S j}^{T}\left(m_{H_{u}}^{2}+m_{\tilde{S}}^{2}\right)+2 A_{S i}^{*} A_{S j}^{T}\right), \\
\left(\beta_{m_{\tilde{d}^{c}}^{2}}^{\mathbf{2 4 , 1}}\right)_{i j}= & 2\left(y_{V i}^{*}\left(\vec{y}_{V}^{T} m_{\tilde{d}^{c}}^{2}\right)_{j}+\left(m_{\tilde{d}^{c}}^{2} \vec{y}_{V}^{*}\right)_{i} y_{V j}^{T}+2 y_{V i}^{*} y_{V j}^{T}\left(m_{H_{u}}^{2}+m_{\tilde{S}}^{2}\right)+2 A_{V i}^{*} A_{V j}^{T}\right), \\
\beta_{m_{H_{u}}^{2}}^{\mathbf{2 4 , 1}}= & 2\left(\frac{3}{2} \vec{y}_{T}^{\dagger} m_{\tilde{L}}^{2} \vec{y}_{T}+\vec{y}_{S}^{\dagger} m_{\tilde{L}}^{2} \vec{y}_{S}+3 \vec{y}_{V}^{\dagger} m_{\tilde{d}_{c}}^{2} \vec{y}_{V}+m_{H_{u}}^{2}\left(\frac{3}{2}\left|\overrightarrow{y_{T}}\right|^{2}+\left|\overrightarrow{y_{S}}\right|^{2}+3\left|\overrightarrow{y_{V}}\right|^{2}\right)+\right. \\
& \left.\frac{3}{2} m_{T}^{2}\left|\overrightarrow{y_{T}}\right|^{2}+m_{S}^{2}\left|\overrightarrow{Y_{S}}\right|^{2}+3 m_{V}^{2}\left|\overrightarrow{y_{V}}\right|^{2}+\frac{3}{2}\left|\overrightarrow{A_{T}}\right|^{2}+\left|\overrightarrow{A_{S}}\right|^{2}+3\left|\overrightarrow{A_{V}}\right|^{2}\right), \\
\beta_{m_{S}^{2}}^{\mathbf{2 4}, 1}= & 4\left(\vec{y}_{S}^{\dagger} m_{\tilde{L}}^{2} \vec{y}_{S}+\left(m_{H_{u}}^{2}+m_{S}^{2}\right)\left|\overrightarrow{y_{S}}\right|^{2}+\left|\overrightarrow{A_{S}}\right|^{2}\right), \\
\beta_{m_{T}^{2}}^{\mathbf{2 4 , 1}}= & 2\left(\vec{y}_{T}^{\dagger} m_{\tilde{L}}^{2} \vec{y}_{T}+\left(m_{H_{u}}^{2}+m_{T}^{2}\right)\left|\overrightarrow{y_{T}}\right|^{2}+\left|\overrightarrow{A_{T}}\right|^{2}\right)-16 M_{2}^{2} g_{2}^{2}, \\
\beta_{m_{V}^{2}}^{\mathbf{2 4}, 1}= & 2\left(\vec{y}_{V}^{\dagger} m_{\tilde{L}}^{2} \vec{y}_{V}+\left(m_{H_{u}}^{2}+m_{V}^{2}\right)\left|\overrightarrow{y_{V}}\right|^{2}+\left|\overrightarrow{A_{V}}\right|^{2}\right)-\frac{10}{3} M_{1}^{2} g_{1}^{2}-6 M_{2}^{2} g_{2}^{2}-\frac{32}{3} M_{3}^{2} g_{3}^{2}-g_{1}^{2} S,
\end{aligned}
$$

while $\left(\beta_{m_{\tilde{Q}}^{24,1}}^{\mathbf{2 4}}\right)_{i j}=\left(\beta_{m_{\tilde{u}^{c}}}^{\mathbf{2 4 , 1}}\right)_{i j}=\left(\beta_{m_{\tilde{e}^{c}}}^{\mathbf{2 4 , 1}}\right)_{i j}=\beta_{m_{H_{d}}^{24,1}}^{\mathbf{2 4}}=0$. The hypercharge D-term contribution $S$ is given by:

$$
S=m_{H_{u}}^{2}-m_{H_{d}}^{2}+\operatorname{Tr}\left(-m_{\tilde{L}^{2}}^{2}+m_{\tilde{e}^{c}}^{2}-2 m_{\tilde{u}^{c}}^{2}+m_{\tilde{d}^{c}}^{2}+m_{\tilde{Q}}^{2}\right)+5\left(m_{V}^{2}-m_{\bar{V}^{2}}^{2}\right) .
$$

Let us finally write the $\beta$-functions for the trilinear terms:

$$
\begin{aligned}
& \left(\beta_{A_{u}}^{\mathbf{2 4 , 1}}\right)_{i j}=\left(\frac{3}{2}\left|\vec{y}_{T}\right|^{2}+\left|\vec{y}_{S}\right|^{2}+3\left|\vec{y}_{V}\right|^{2}\right)\left(A_{u}\right)_{i j}+\left(3 \vec{y}_{T} \cdot \vec{A}_{T}+2 \vec{y}_{S} \cdot \vec{A}_{S}+6 \vec{y}_{V} \cdot \vec{A}_{V}\right)\left(Y_{u}\right)_{i j}, \\
& \left(\beta_{A_{d}}^{\mathbf{2 4}, 1}\right)_{i j}=2 y_{V i}\left(\vec{y}_{V}^{\dagger} A_{d}\right)_{j}+4 A_{V i}\left(\vec{y}_{V}^{\dagger} Y_{d}\right)_{j} \\
& \left(\beta_{A_{e}}^{\mathbf{2 4}, 1}\right)_{i j}=\frac{3}{2}\left(A_{e} \vec{y}_{T}^{*}\right)_{i} y_{T j}^{T}+\left(A_{e} \vec{y}_{S}^{*}\right)_{i} y_{S j}^{T}+3\left(Y_{e} \vec{y}_{T}^{*}\right)_{i} A_{T j}^{T}+2\left(Y_{e} \vec{y}_{S}^{*}\right)_{i} A_{S j}^{T}, \\
& \left(\beta_{A_{S}}^{\mathbf{2 4 , 1}}\right)_{i}=-\frac{3}{5}\left(A_{S i}-2 M_{1} y_{S i}\right) g_{1}^{2}-3\left(A_{S i}-2 M_{2} y_{S i}\right) g_{2}^{2}+\left(3 \operatorname{Tr}\left(Y_{u} Y_{u}^{\dagger}\right)+\frac{3}{2}\left|\vec{y}_{T}\right|^{2}+5\left|\vec{y}_{S}\right|^{2}+3\left|\vec{y}_{V}\right|^{2}\right) A_{S i}+ \\
& \left(6 \operatorname{Tr}\left(A_{u} Y_{u}^{\dagger}\right)+3 \vec{y}_{T}^{*} \cdot \vec{A}_{T}+7 \vec{y}_{S}^{*} \cdot \vec{A}_{S}+6 \vec{y}_{V}^{*} \cdot \vec{A}_{V}\right) y_{S i}+ \\
& \left(Y_{e}^{T} Y_{e}^{*} \vec{A}_{S}\right)_{i}+2\left(A_{e}^{T} Y_{e}^{*} \vec{y}_{S}\right)_{i}+\frac{3}{2}\left(\vec{y}_{T}^{*} \cdot \vec{y}_{S}\right) A_{T i}+3\left(\vec{y}_{T}^{*} \cdot \vec{A}_{S}\right) y_{T i}, \\
& \left(\beta_{A_{T}}^{\mathbf{2 4 , 1}}\right)_{i}=-\frac{3}{5}\left(A_{T i}-2 M_{1} y_{T i}\right) g_{1}^{2}-7\left(A_{T i}-2 M_{2} y_{T i}\right) g_{2}^{2}+\left(3 \operatorname{Tr}\left(Y_{u} Y_{u}^{\dagger}\right)+\frac{5}{2}\left|\vec{y}_{T}\right|^{2}+\left|\vec{y}_{S}\right|^{2}+3\left|\vec{y}_{V}\right|^{2}\right) A_{T i}+ \\
& \left(6 \operatorname{Tr}\left(A_{u} Y_{u}^{\dagger}\right)+\frac{13}{2} \vec{y}_{T}^{*} \cdot \vec{A}_{T}+2 \vec{y}_{S}^{*} \cdot \vec{A}_{S}+6 \vec{y}_{V}^{*} \cdot \vec{A}_{V}\right) y_{T i}+ \\
& \left(Y_{e}^{T} Y_{e}^{*} \vec{A}_{T}\right)_{i}+2\left(A_{e}^{T} Y_{e}^{*} \vec{y}_{T}\right)_{i}+\left(\vec{y}_{S}^{*} \cdot \vec{y}_{T}\right) A_{S i}+2\left(\vec{y}_{S}^{*} \cdot \vec{A}_{T}\right) y_{S i} \\
& \left(\beta_{A_{V}}^{\mathbf{2 4}, 1}\right)_{i}=-\frac{19}{15}\left(A_{V i}-2 M_{1} y_{V i}\right) g_{1}^{2}-3\left(A_{V i}-2 M_{2} y_{V i}\right) g_{2}^{2}-\frac{16}{3}\left(A_{V i}-2 M_{1} y_{V i}\right) g_{3}^{2}+ \\
& \left(3 \operatorname{Tr}\left(Y_{u} Y_{u}^{\dagger}\right)+\frac{3}{2}\left|\vec{y}_{T}\right|^{2}+\left|\vec{y}_{S}\right|^{2}+8\left|\vec{y}_{V}\right|^{2}\right) A_{V i}+\left(3 \operatorname{Tr}\left(A_{u} Y_{u}^{\dagger}\right)+3 \vec{y}_{T}^{*} \cdot \vec{A}_{T}+2 \vec{y}_{S}^{*} \cdot \vec{A}_{S}+11 \vec{y}_{V}^{*} \cdot \vec{A}_{V}\right) y_{V i}+ \\
& 2\left(Y_{d} Y_{d}^{\dagger} \vec{A}_{V}\right)_{i}+4\left(A_{d} Y_{d}^{\dagger} \vec{y}_{V}\right)_{i}
\end{aligned}
$$

[1] P. Minkowski, Phys. Lett. B 67421 (1977); M. GellMann, P. Ramond and R. Slansky, in Supergravity, edited by P. van Nieuwenhuizen and D. Freedman, (North- 
Holland, 1979), p. 315; T. Yanagida, in Proceedings of the Workshop on the Unified Theory and the Baryon Number in the Universe, edited by O. Sawada and A. Sugamoto (KEK Report No. 79-18, Tsukuba, 1979), p. 95; R.N. Mohapatra and G. Senjanović, Phys. Rev. Lett. 44 (1980) 912.

[2] M. Magg and C. Wetterich, Phys. Lett. B 94, 61 (1980); J. Schechter and J. W. F. Valle, Phys. Rev. D 23 (1981) 1666; C. Wetterich, Nucl. Phys. B 187, 343 (1981); G. Lazarides, Q. Shafi and C. Wetterich, Nucl. Phys. B 181, 287 (1981); R. N. Mohapatra and G. Senjanovic, Phys. Rev. D 23, 165 (1981).

[3] R. Foot, H. Lew, X. G. He and G. C. Joshi, Z. Phys. C 44, 441 (1989).

[4] A. Abada, C. Biggio, F. Bonnet, M. B. Gavela and T. Hambye, JHEP 0712 (2007) 061 arXiv:0707.4058 [hep-ph]].

[5] E. Ma, Phys. Rev. Lett. $81 \quad$ (1998) 1171 arXiv:hep-ph/9805219.

[6] P. Fileviez Perez, Phys. Lett. B 654 (2007) 189 arXiv:hep-ph/0702287; P. Fileviez Perez, Phys. Rev. D 76, 071701 (2007) arXiv:0705.3589 [hep-ph]].

[7] P. Fileviez Perez, H. Iminniyaz, G. Rodrigo and S. Spinner, Phys. Rev. D 81 (2010) 095013 arXiv:0911.1360 [hep-ph]].

[8] I. K. Cooper, S. F. King and C. Luhn, Phys. Lett. B 690 (2010) 396 arXiv:1004.3243 [hep-ph]].

[9] F. Borzumati and T. Yamashita, arXiv:0903.2793 [hep$\mathrm{ph}$.

[10] H. Georgi and S. L. Glashow, Phys. Rev. Lett. 32 (1974) 438.

[11] A. Rossi, Phys. Rev. D 66, 075003 (2002) arXiv:hep-ph/0207006.

[12] B. Bajc and G. Senjanovic, JHEP 0708 (2007) 014 arXiv:hep-ph/0612029.

[13] B. Bajc, M. Nemevsek and G. Senjanovic, Phys. Rev. D 76 (2007) 055011 arXiv:hep-ph/0703080.

[14] A. Abada, C. Biggio, F. Bonnet, M. B. Gavela and T. Hambye, Phys. Rev. D 78 (2008) 033007 arXiv:0803.0481 [hep-ph]].

[15] J. F. Kamenik and M. Nemevsek, JHEP 0911 (2009) 023 arXiv:0908.3451 [hep-ph]].

[16] J. R. Ellis and M. K. Gaillard, Phys. Lett. B 88 (1979) 315.

[17] H. Georgi and C. Jarlskog, Phys. Lett. B 86 (1979) 297.

[18] A. Ibarra and G. G. Ross, Phys. Lett. B 591 (2004) 285 arXiv:hep-ph/0312138.

[19] A. Ibarra, JHEP $0601 \quad$ (2006) 064 arXiv:hep-ph/0511136.

[20] W. 1. Guo, Z. z. Xing and S. Zhou, Int. J. Mod. Phys. E 16 (2007) 1 arXiv:hep-ph/0612033.

[21] M. C. Gonzalez-Garcia, M. Maltoni and J. Salvado, JHEP 1004 (2010) 056 arXiv:1001.4524 [hep-ph]].

[22] F. Borzumati and A. Masiero, Phys. Rev. Lett. 57 (1986) 961.

[23] I. Masina and C. A. Savoy, Nucl. Phys. B 661 (2003) 365 arXiv:hep-ph/0211283; P. Paradisi, JHEP 0510 (2005) 006 arXiv:hep-ph/0505046.

[24] F. R. Joaquim, JHEP 1006 (2010) 079 arXiv:0912.3427 [hep-ph]].

[25] W. Altmannshofer, A. J. Buras, S. Gori, P. Paradisi and D. M. Straub, Nucl. Phys. B 830 (2010) 17 arXiv:0909.1333 [hep-ph]].

[26] J. Hisano, T. Moroi, K. Tobe and M. Yamaguchi, Phys.
Rev. D 53 (1996) 2442 arXiv:hep-ph/9510309; T. Moroi, Phys. Rev. D 53 (1996) 6565 Erratum-ibid. D 56 (1997) 4424] arXiv:hep-ph/9512396.

[27] M. Ciuchini et al., JHEP 9810, 008 (1998) arXiv:hep-ph/9808328; D. Becirevic et al., Nucl. Phys. B 634, 105 (2002) arXiv:hep-ph/0112303.

[28] P. Gondolo, J. Edsjo, P. Ullio, L. Bergstrom, M. Schelke and E. A. Baltz, JCAP 0407 (2004) 008 arXiv:astro-ph/0406204.

[29] G. Degrassi, P. Gambino and P. Slavich, Comput. Phys. Commun. 179 (2008) 759 arXiv:0712.3265 [hep-ph]].

[30] E. Barberio et al. [Heavy Flavor Averaging Group], arXiv:0808.1297 [hep-ex].

[31] M. L. Brooks et al. [MEGA Collaboration], Phys. Rev. Lett. 83, $1521 \quad$ (1999) arXiv:hep-ex/9905013; M. Ahmed et al. [MEGA Collaboration], Phys. Rev. D 65, 112002 (2002) arXiv:hep-ex/0111030.

[32] L. M. Barkov et al., PSI Proposal R-99-05 (1999); S. Ritt [MEG Collaboration], Nucl. Phys. Proc. Suppl. 162, 279 (2006); J. Adam et al. [MEG collaboration], Nucl. Phys. B 834 (2010) 1 arXiv:0908.2594 [hep-ex]].

[33] M. Bona et al., arXiv:0709.0451 [hep-ex].

[34] A. G. Akeroyd et al. [SuperKEKB Physics Working Group], arXiv:hep-ex/0406071.

[35] T. Aaltonen et al. [CDF Collaboration], Phys. Rev. Lett. 100 (2008) 161802 arXiv:0712.2397 [hep-ex]].

[36] V. M. Abazov et al. [D0 Collaboration], Phys. Rev. Lett. 101 (2008) 241801 arXiv:0802.2255 [hep-ex]].

[37] V. M. Abazov et al. [D0 Collaboration], arXiv:1005.2757 [hep-ex]; V. M. Abazov et al. [D0 Collaboration], arXiv:1007.0395 [hep-ex].

[38] A. J. Buras and D. Guadagnoli, Phys. Rev. D 78 (2008) 033005 arXiv:0805.3887 [hep-ph]]; A. J. Buras and D. Guadagnoli, Phys. Rev. D 79 (2009) 053010 arXiv:0901.2056 [hep-ph]].

[39] E. Lunghi and A. Soni, Phys. Lett. B 666 (2008) 162 arXiv:0803.4340 [hep-ph]].

[40] J. Dunkley et al. [WMAP Collaboration], Astrophys. J. Suppl. 180 (2009) 306 arXiv:0803.0586 [astro-ph]]; E. Komatsu et al. [WMAP Collaboration], Astrophys. J. Suppl. 180 (2009) 330 arXiv:0803.0547 [astro-ph]].

[41] J. R. Ellis, T. Falk and K. A. Olive, Phys. Lett. B 444, 367 (1998) arXiv:hep-ph/9810360; J. R. Ellis, T. Falk, K. A. Olive and M. Srednicki, Astropart. Phys. 13, 181 (2000) [Erratum-ibid. 15, 413 (2001)] arXiv:hep-ph/9905481.

[42] K. L. Chan, U. Chattopadhyay and P. Nath, Phys. Rev. D 58, 096004 (1998) arXiv:hep-ph/9710473; J. L. Feng, K. T. Matchev and T. Moroi, Phys. Rev. Lett. 84, 2322 (2000) arXiv:hep-ph/9908309 ; J. L. Feng, K. T. Matchev and F. Wilczek, Phys. Lett. B 482, 388 (2000) arXiv:hep-ph/0004043.

[43] M. Drees and M. M. Nojiri, Phys. Rev. D 47, 376 (1993) arXiv:hep-ph/9207234.

[44] L. Calibbi, M. Frigerio, S. Lavignac and A. Romanino, JHEP 0912 (2009) 057 arXiv:0910.0377 [hep-ph]].

[45] L. Calibbi, L. Ferretti, A. Romanino and R. Ziegler, JHEP 0903 (2009) 031 arXiv:0812.0087 [hep-ph]].

[46] J. Ellis, A. Mustafayev and K. A. Olive, arXiv:1003.3677 [hep-ph]; J. Ellis, A. Mustafayev and K. A. Olive, arXiv:1004.5399 [hep-ph].

[47] L. Calibbi, Y. Mambrini and S. K. Vempati, JHEP 0709 (2007) 081 arXiv:0704.3518 [hep-ph]]. 
[48] M. Drees and J. M. Kim, JHEP 0812 (2008) 095 arXiv:0810.1875 [hep-ph]].

[49] E. Carquin, J. Ellis, M. E. Gomez, S. Lola and J. Rodriguez-Quintero, JHEP 0905 (2009) 026 arXiv:0812.4243 [hep-ph]].

[50] M. E. Gomez, S. Lola, P. Naranjo and J. RodriguezQuintero, JHEP 1006 (2010) 053 [arXiv:1003.4937 [hep$\mathrm{ph}]$.

[51] B. Grinstein, Nucl. Phys. B 206 (1982) 387; A. Masiero, D. V. Nanopoulos, K. Tamvakis and T. Yanagida, Phys. Lett. B 115 (1982) 380.
[52] G. Altarelli, F. Feruglio and I. Masina, JHEP 0011 (2000) 040 arXiv:hep-ph/0007254.

[53] P. Nath and P. Fileviez Perez, Phys. Rept. 441 (2007) 191 arXiv:hep-ph/0601023.

[54] S. Blanchet and P. Fileviez Perez, JCAP 0808 (2008) 037 arXiv:0807.3740 [hep-ph]].

[55] S. P. Martin and M. T. Vaughn, Phys. Rev. D 50 (1994) 2282 [Erratum-ibid. D 78 (2008) 039903 ] arXiv:hep-ph/9311340. 